\title{
Complexity of Existential Positive First-Order Logic
}

\author{
Manuel Bodirsky, Miki Hermann \\ LIX (UMR CNRS 7161), École Polytechnique, 91128 Palaiseau, France \\ \{bodirsky, hermann\}@lix.polytechnique.fr \\ Florian Richoux* \\ JFLI, CNRS - University of Tokyo, Japan \\ richoux@jfli.itc.u-tokyo.ac.jp
}

\begin{abstract}
Let $\Gamma$ be a (not necessarily finite) structure with a finite relational signature. We prove that deciding whether a given existential positive sentence holds in $\Gamma$ is in LOGSPACE or complete for the class $\operatorname{CSP}(\Gamma)_{\mathrm{NP}}$ under deterministic polynomial-time many-one reductions. Here, $\operatorname{CSP}(\Gamma)_{\mathrm{NP}}$ is the class of problems that can be reduced to the constraint satisfaction problem of $\Gamma$ under non-deterministic polynomial-time many-one reductions.

Key words: Computational Complexity, Existential Positive First-Order Logic, Constraint Satisfaction Problems
\end{abstract}

\section{Introduction}

We study the computational complexity of the following class of computational problems. Let $\Gamma$ be a structure with finite or infinite domain and with a finite relational signature. The model-checking problem for existential positive first-order logic, parametrized by $\Gamma$, is the following problem.

Problem: $\operatorname{ExPOS}(\Gamma)$

Input: An existential positive first-order sentence $\Phi$.

Question: Does $\Gamma$ satisfy $\Phi$ ?

Existential positive first-order formula over $\Gamma$ are first-order formulas without universal quantifiers, equalities, and negation symbols, and formally defined as follows:

- if $R$ is a relation symbol of a relation from $\Gamma$ with arity $k$ and $x_{1}, \ldots, x_{k}$ are (not necessarily distinct) variables, then $R\left(x_{1}, \ldots, x_{k}\right)$ is an existential positive first-order formula (such formulas are called atomic);

- if $\varphi$ and $\psi$ are existential positive first-order formulas, then $\varphi \wedge \psi$ and $\varphi \vee \psi$ are existential positive first-order formulas;

- if $\varphi$ is an existential positive first-order formula with a free variable $x$ then $\exists x . \varphi$ is an existential positive first-order formula.

\footnotetext{
${ }^{*}$ This work was done during the PhD studies of the third author at École Polytechnique.
} 
An existential positive first-order sentence is an existential positive first-order formula without free variables.

Note that we do not allow the equality symbol in the existential positive sentences; this only makes our results stronger, since one might always add a relation symbol $=$ for the equality relation to the signature of $\Gamma$ to obtain the result for the case where the equality symbol is allowed. Also note that adding a symbol for equality to $\Gamma$ might change the complexity of $\operatorname{ExPOS}(\Gamma)$. Consider for example $\Gamma:=(\mathbb{N} ; \neq)$; here, $\operatorname{ExPOS}(\Gamma)$ can be reduced to the Boolean formula evaluation problem (which is known to be in LOGSPACE) as follows: atomic formulas in $\Phi$ of the form $x \neq y$ are replaced by true, and atomic formulas of the form $x \neq x$ are replaced by false. The resulting Boolean formula is equivalent to true if and only if $\Phi$ is true in $\Gamma$. However, the problem $\operatorname{ExPos}\left(\Gamma^{\prime}\right)$ for $\Gamma^{\prime}:=(\mathbb{N} ; \neq,=)$ is NP-complete. Similar examples exist over finite domains.

The constraint satisfaction problem $\operatorname{CSP}(\Gamma)$ for $\Gamma$ is defined similarly, but its input consists of a primitive positive sentence, that is, a existential positive sentence without disjunctions. Constraint satisfaction problems frequently appear in many areas of computer science, and have attracted a lot of attention, in particular in combinatorics, artificial intelligence, finite model theory and universal algebra; we refer to the recent collection of survey articles on this subject [1]. The class of constraint satisfaction problems for infinite structures $\Gamma$ is a rich class of problems; it can be shown that for every computational problem there exists a relational structure $\Gamma$ such that $\operatorname{CSP}(\Gamma)$ is equivalent to that problem under polynomial-time Turing reductions [2].

In this paper, we show that the complexity classification for existential positive first-order sentences over infinite structures can be reduced to the complexity classification for constraint satisfaction problems. For finite structures $\Gamma$, our result implies that $\operatorname{ExPOS}(\Gamma)$ is in LOGSPACE or NP-complete. The LOGSPACEsolvable cases of $\operatorname{ExPOS}(\Gamma)$ are in this case precisely those relational structures $\Gamma$ with an element $a$ such that all non-empty relations in $\Gamma$ contain the tuple $(a, \ldots, a)$; in this case, $\operatorname{ExPOS}(\Gamma)$ is called a-valid. Interestingly, this is no longer true for infinite structures $\Gamma$. To see this, consider again the structure $\Gamma:=$ $(\mathbb{N} ; \neq)$, which is clearly not $a$-valid, but in LOGSPACE as we have noticed above.

A universal-algebraic study of the model-checking problem for finite structures $\Gamma$ and various other syntactic restrictions of first-order logic (for instance positive first-order logic) can be found in [9].

A preliminary version of this article appeared in [3]. The present version differs in that the main proof has been simplified and now also works without the relation symbol for equality; moreover, Proposition 3 and Section 4 have been added.

\section{Main Result}

We write $L \leq_{m} L^{\prime}$ if there exists a deterministic polynomial-time many-one reduction from $L$ to $L^{\prime}$.

Definition 1 (from [6]) A problem $A$ is non-deterministic polynomial-time many-one reducible to a problem $B\left(A \leq_{\mathrm{NP}} B\right)$ if there is a nondeterministic polynomial-time Turing machine $M$ such that $x \in A$ if and only if there exists a computation of $M$ that outputs $y$ on input $x$, and $y \in B$. We denote by $A_{\mathrm{NP}}$ the smallest class that contains $A$ and is downward closed under $\leq_{\mathrm{NP}}$.

Observe that $\leq_{\mathrm{NP}}$ is transitive [6]. To state the complexity classification for existential positive firstorder logic, we need the following concept. The $\Gamma$-localizer $F(\psi)$ of a formula $\psi$ is defined as follows:

- $F(\exists x . \psi)=F(\psi)$

- $F(\varphi \wedge \psi)=F(\varphi) \wedge F(\psi)$

- $F(\varphi \vee \psi)=F(\varphi) \vee F(\psi)$ 
- When $\psi$ is atomic, then $F(\psi)= \begin{cases}\text { true } & \text { if } \psi \text { is satisfiable in } \Gamma \\ \text { false } & \text { otherwise }\end{cases}$

Definition 2 We call a structure $\Gamma$ locally refutable if every existential positive sentence $\Phi$ is true in $\Gamma$ if and only if the $\Gamma$-localizer $F(\Phi)$ is logically equivalent to true.

Proposition 3 A structure $\Gamma$ is locally refutable if and only if every unsatisfiable conjunction of atomic formulas contains an unsatisfiable conjunct.

Proof: First suppose that $\Gamma$ is locally refutable, and let $\varphi$ be a conjunction of atomic formulas with variables $x_{1}, \ldots, x_{n}$. Then every conjunct of $\varphi$ is satisfiable in $\Gamma$ if and only if $F(\varphi)$ is true. By local refutability of $\Gamma$ this is the case if and only if $\exists x_{1}, \ldots, x_{n} . \varphi$ is true in $\Gamma$, which shows the claim.

Now suppose that $\Gamma$ is not locally refutable, that is, there is an existential positive sentence $\Phi$ that is false in $\Gamma$ such that $F(\Phi)$ is true. Define recursively for each subformula $\psi$ of $\Phi$ where $F(\psi)$ is true the formula $T(\psi)$ as follows. If $\psi$ is of the form $\psi_{1} \vee \psi_{2}$, then for some $i \in\{1,2\}$ the formula $F\left(\psi_{i}\right)$ must be true, and we set $T(\psi)$ to be $T\left(\psi_{i}\right)$. If $\psi$ is of the form $\psi_{1} \wedge \psi_{2}$, then for both $i \in\{1,2\}$ the formula $F\left(\psi_{i}\right)$ must be true, and we set $T(\Psi)$ to be $T\left(\psi_{1}\right) \wedge T\left(\psi_{2}\right)$.

Each conjunct $\varphi$ in $T(\Phi)$ is satisfiable in $\Gamma$ since $F(\Phi)$ is true. But since $\Phi$ is false in $\Gamma, T(\Phi)$ must be unsatisfiable.

In Section 3, we will show the following result.

Theorem 4 Let $\Gamma$ be a structure with a finite relational signature $\tau$. If $\Gamma$ is locally refutable then the problem $\operatorname{ExPOS}(\Gamma)$ to decide whether an existential positive sentence is true in $\Gamma$ is in LOGSPACE. If $\Gamma$ is not locally refutable, then $\operatorname{ExPOS}(\Gamma)$ is complete for the class $\operatorname{CSP}(\Gamma)_{\mathrm{NP}}$ under polynomial-time many-one reductions.

In particular, $\operatorname{ExPOS}(\Gamma)$ is in LOGSPACE or is NP-hard (under deterministic polynomial-time manyone reductions). If $\Gamma$ is finite, then $\operatorname{ExPOS}(\Gamma)$ is in LOGSPACE or NP-complete, because finite domain constraint satisfaction problems are clearly in NP. The observation that $\operatorname{ExPOS}(\Gamma)$ is in LOGSPACE or NP-complete has previously been made in [5] and independently in [8]. However, our proof remains the same for finite domains and is simpler than the previous proofs.

\section{Proof}

Before we prove Theorem 4, we start with the following simpler result.

Theorem 5 Let $\Gamma$ be a structure with a finite relational signature $\tau$. If $\Gamma$ is locally refutable, then the problem $\operatorname{ExPOS}(\Gamma)$ to decide whether an existential positive sentence is true in $\Gamma$ is in LOGSPACE. If $\Gamma$ is not locally refutable, then $\operatorname{ExPOS}(\Gamma)$ is NP-hard (under polynomial-time many-one reductions).

To prove Theorem 5, we need first to prove the following lemma.

Lemma 6 A structure $\Gamma$ is not locally refutable if and only if there are existential positive formulas $\psi_{0}$ and $\psi_{1}$ with the property that

- $\psi_{0}$ and $\psi_{1}$ define non-empty relations over $\Gamma$;

- $\psi_{0} \wedge \psi_{1}$ defines the empty relation over $\Gamma$. 
Proof: The "if"-part of the statement is immediate. To show the "only if"-part, suppose that $\Gamma$ is not locally refutable. Then by Proposition 3 there is an unsatisfiable conjunction $\psi$ of satisfiable atomic formulas. Among all such formulas $\psi$, let $\psi$ be one of minimal length. Let $\psi_{0}$ be one of the atomic formulas in $\psi$, and let $\psi_{1}$ be the conjunction over the remaining conjuncts in $\psi$. Since $\psi$ was chosen to be minimal, the formula $\psi_{1}$ must be satisfiable. By construction $\psi_{0}$ is also satisfiable and $\psi$ is unsatisfiable, which is what we had to show.

Proof of Theorem 5: If $\Gamma$ is locally refutable, then $\operatorname{ExPOS}(\Gamma)$ can be reduced to the positive Boolean formula evaluation problem, which is known to be LOGSPACE-complete. We only have to construct from an existential positive sentence $\Phi$ a Boolean formula $F:=F_{\Gamma}(\Phi)$ as described before Definition 2 . Clearly, this construction can be performed with logarithmic work-space. We evaluate $F$, and reject if $F$ is false, and accept otherwise.

If $\Gamma$ is not locally refutable, we show NP-hardness of $\operatorname{ExPOS}(\Gamma)$ by reduction from 3-SAT. Let $I$ be a 3-SAT instance. We construct an instance $\Phi$ of $\operatorname{ExPOS}(\Gamma)$ as follows. Let $\psi_{0}$ and $\psi_{1}$ be the formulas from Lemma6 (suppose they are $d$-ary). Let $v_{1}, \ldots, v_{n}$ be the Boolean variables in $I$. For each $v_{i}$ we introduce $d$ new variables $\bar{x}_{i}=x_{i}^{1}, \ldots, x_{i}^{d}$. Let $\Phi$ be the instance of $\operatorname{ExPOS}(\Gamma)$ that contains the following conjuncts:

- For each $1 \leq i \leq n$, the formula $\psi_{0}\left(\bar{x}_{i}\right) \vee \psi_{1}\left(\bar{x}_{i}\right)$

- For each clause $l_{1} \vee l_{2} \vee l_{3}$ in $I$, the formula $\psi_{i_{1}}\left(\bar{x}_{j_{1}}\right) \vee \psi_{i_{2}}\left(\bar{x}_{j_{2}}\right) \vee \psi_{i_{3}}\left(\bar{x}_{j_{3}}\right)$ where $i_{p}=0$ if $l_{p}$ equals $\neg x_{j_{p}}$ and $i_{p}=1$ if $l_{p}$ equals $x_{j_{p}}$, for all $p \in\{1,2,3\}$.

It is clear that $\Phi$ can be computed in deterministic polynomial time from $I$, and that $\Phi$ is true in $\Gamma$ if and only if $I$ is satisfiable.

Applied to finite relational structures $\Gamma$, we obtain the result from [5] and [8], that is, $\operatorname{ExPos}(\Gamma)$ is in LOGSPACE if $\Gamma$ is $a$-valid and NP-complete otherwise. We prove in the following proposition that, over a finite domain $D, \Gamma$ is locally refutable if and only if it is $a$-valid for an element $a \in D$.

Proposition 7 Let $\Gamma$ be a relational structure with a finite domain $D$. Then $\Gamma$ is locally refutable if and only if it is a-valid for an element $a \in D$.

Proof: Suppose that $\Gamma$ is $a$-valid, and let $\Phi$ be an existential positive sentence over the signature of $\Gamma$. To show that $\Gamma$ is locally refutable, we only have to show that $\Phi$ is true in $\Gamma$ when $F(\Phi)$ is equivalent to true (since the other direction holds trivially). But this follows from the fact that if an atomic formula $R\left(x_{1}, \ldots, x_{n}\right)$ is satisfiable in $\Gamma$ then in fact this formula can be satisfied by setting all variables to $a$.

For the opposite direction of the statement, let $D=\left\{a_{1}, \ldots, a_{n}\right\}$, and suppose that for all $a \in D$ the structure $\Gamma$ is not $a$-valid. That is, for each $a_{i} \in D$ there exists a non-empty relation $R_{i}$ of arity $r_{i}$ in $\Gamma$ such that $\left(a_{i}, \ldots, a_{i}\right) \notin R$. Let $r$ be $\sum_{i=1}^{n} r_{i}$, and let $x_{1}, \ldots, x_{r n}$ be distinct variables. Consider the formula

$$
\psi=\bigwedge_{\bar{y} \in\left\{x_{1}, \ldots, x_{r n}\right\}^{r}} R_{1}\left(y_{1}, \ldots, y_{r_{1}}\right) \wedge \cdots \wedge R_{n}\left(y_{r-r_{n}+1}, \ldots, y_{r}\right)
$$

By the pigeonhole principle, for every mapping $f:\left\{x_{1}, \ldots, x_{r n}\right\} \rightarrow D$ at least $r$ variables are mapped to the same value, say to $a_{i}$. For a vector $\bar{y}$ that contains exactly these $r$ variables, for some $l$ there is a conjunct $R_{i}\left(y_{l+1}, \ldots, y_{l+r_{i}}\right)$ in $\psi$; but by assumption, $R_{i}$ does not contain the tuple $\left(a_{i}, \ldots, a_{i}\right)$. This shows that $\exists x_{1}, \ldots, x_{r n} . \psi$ is not true in $\Gamma$. On the other hand, since each relation $R_{i}$ is non-empty, it is clear that the Boolean formula $F\left(\exists x_{1}, \ldots, x_{r n} . \psi\right)$ is true. Therefore, $\Gamma$ is not locally refutable. 
Remark 8 In the proof of Theorem 4 it will be convenient to assume that $\Gamma$ has a single relation $R$. When we study the problem $\operatorname{CSP}(\Gamma)$, this is without loss of generality, since we can always find a CSP which is deterministic polynomial-time equivalent and where the template is of this form: if $\Gamma=\left(D ; R_{1}, \ldots, R_{n}\right)$ where $R_{i}$ has arity $r_{i}$ and is not empty, then $\operatorname{CSP}(\Gamma)$ is equivalent to $\operatorname{CSP}\left(D ; R_{1} \times \cdots \times R_{n}\right)$ where $R_{1} \times \cdots \times R_{n}$ is the $\sum_{i=1}^{n} r_{i}$-ary relation defined as the Cartesian product of the relations $R_{1}, \ldots, R_{n}$. Similarly, $\operatorname{ExPOS}(\Gamma)$ is equivalent to $\operatorname{ExPOS}\left(D ; R_{1} \times \cdots \times R_{n}\right)$.

Proof of Theorem 4. If $\Gamma$ is locally refutable then the statement has been shown in Theorem 5. Suppose that $\Gamma$ is not locally refutable. To show that $\operatorname{ExPos}(\Gamma)$ is contained in $\operatorname{CSP}(\Gamma)_{\mathrm{NP}}$, we construct a nondeterministic Turing machine $T$ which takes as input an instance $\Phi$ of $\operatorname{ExPOS}(\Gamma)$, and which outputs an instance $T(\Phi)$ of $\operatorname{CSP}(\Gamma)$ as follows.

On input $\Phi$ the machine $T$ proceeds recursively as follows:

- if $\Phi$ is of the form $\exists x . \varphi$ then return $\exists x . T(\varphi)$;

- if $\Phi$ is of the form $\varphi_{1} \wedge \varphi_{2}$ then return $T\left(\varphi_{1}\right) \wedge T\left(\varphi_{2}\right)$;

- if $\Phi$ is of the form $\varphi_{1} \vee \varphi_{2}$ then non-deterministically return either $T\left(\varphi_{1}\right)$ or $T\left(\varphi_{2}\right)$;

- if $\Phi$ is of the form $R\left(x_{1}, \ldots, x_{k}\right)$ then return $R\left(x_{1}, \ldots, x_{k}\right)$.

The output of $T$ can be viewed as an instance of $\operatorname{CSP}(\Gamma)$, since it can be transformed to a primitive positive sentence (by moving all existential quantifiers to the front). It is clear that $T$ has polynomial running time, and that $\Phi$ is true in $\Gamma$ if and only if there exists a computation of $T$ on $\Phi$ that computes a sentence that is true in $\Gamma$.

We now show that $\operatorname{ExPOS}(\Gamma)$ is hard for $\operatorname{CSP}(\Gamma)_{\mathrm{NP}}$ under $\leq_{m}$-reductions. Let $L$ be a problem with a non-deterministic polynomial-time many-one reduction to $\operatorname{CSP}(\Gamma)$, and let $M$ be the non-deterministic Turing machine that computes the reduction. We have to construct a deterministic Turing machine $M^{\prime}$ that computes for any input string $s$ in polynomial time in $|s|$ an instance $\Phi$ of $\operatorname{ExPOS}(\Gamma)$ such that $\Phi$ is true in $\Gamma$ if and only if there exists a computation of $M$ on $s$ that computes a satisfiable instance of $\operatorname{CSP}(\Gamma)$.

Say that the running time of $M$ on $s$ is in $O\left(|s|^{e}\right)$ for a constant $e$. Hence, there are constants $s_{0}$ and $c$ such that for $|s|>s_{0}$ the running time of $M$ and hence also the number of constraints in the input instance of $\operatorname{CSP}(\Gamma)$ produced by the reduction is bounded by $t:=c|s|^{e}$. The non-deterministic computation of $M$ can be viewed as a deterministic computation with access to non-deterministic advice bits as shown in [4]. We also know that for $|s|>s_{0}$, the machine $M$ can access at most $t$ non-deterministic bits. If $w$ is a sufficiently long bit-string, we write $M_{w}$ for the deterministic Turing machine obtained from $M$ by using the bits in $w$ as the non-deterministic bits, and $M_{w}(s)$ for the instance of $\operatorname{CSP}(\Gamma)$ computed by $M_{w}$ on input $s$.

If $|s| \leq s_{0}$, then $M^{\prime}$ returns $\exists \bar{x} \cdot \psi_{1}(\bar{x})$ if there is an $w \in\{0,1\}^{*}$ such that $M_{w}(s)$ is a satisfiable instance of $\operatorname{CSP}(\Gamma)$, and $M^{\prime}$ returns $\exists \bar{x}\left(\psi_{0}(\bar{x}) \wedge \psi_{1}(\bar{x})\right)$ otherwise (i.e., it returns a false instance of $\operatorname{ExPOS}(\Gamma) ; \psi_{0}$ and $\psi_{1}$ are defined in Lemma 6). Since $s_{0}$ is a fixed finite value, $M^{\prime}$ can perform these computations in constant time.

By Remark 8 made above, we can assume without loss of generality that $\Gamma$ has just a single relation $R$. Let $l$ be the arity of $R$. Then instances of $\operatorname{CSP}(\Gamma)$ with variables $x_{1}, \ldots, x_{n}$ can be encoded as sequences of numbers that are represented by binary strings of length $\lceil\log t\rceil$ as follows: the $i$-th number $m$ in this sequence indicates that the $(((i-1) \bmod l)+1)$-st variable in the $(((i-1)$ div $l)+1)$-st constraint is $x_{m}$.

For $|s|>s_{0}$, we use a construction from the proof of Cook's theorem given in [4]. In this proof, a computation of a non-deterministic Turing machine $T$ accepting a language $L$ is encoded by Boolean variables that represent the state and the position of the read-write head of $T$ at time $r$, and the content of 
the tape at position $j$ at time $r$. The tape content at time 0 consists of the input $x$, written at positions 1 through $n$, and the non-deterministic advice bit string $w$, written at positions -1 through $-|w|$. The proof in [4] specifies a deterministic polynomial-time computable transformation $f_{L}$ that computes for a given string $s$ a SAT instance $f_{L}(s)$ such that there is an accepting computation of $T$ on $s$ if and only if there is a satisfying truth assignment for $f_{L}(s)$.

In our case, the machine $M$ computes a reduction and thus computes an output string. Recall our binary representation of instances of the CSP $M$ writes on the output tape a sequence of numbers represented by binary strings of length $\lceil\log t\rceil$. It is straightforward to modify the transformation $f_{L}$ given in the proof of Theorem 2.1 in [4] to obtain for all positive integers $a, b, c$ where $a \leq t, b \leq l, c \leq\lceil\log t\rceil$, and $d \in\{0,1\}$, a deterministic polynomial-time transformation $g_{a, b, c}^{d}$ that computes for a given string $s$ a SAT instance $g_{a, b, c}^{d}(s)$ with distinguished variables $z_{1}, \ldots, z_{p}, p \leq t$ for the non-deterministic bits in the computation of $M$ such that the following are equivalent:

- $g_{a, b, c}^{d}(s)$ has a satisfying assignment where $z_{i}$ is set to $w_{i} \in\{0,1\}$ for $1 \leq i \leq p$;

- the $c$-th bit in the $b$-th variable of the $a$-th constraint in $M_{w}(s)$ equals $d$.

We use the transformations $g_{a, b, c}^{d}$ to define $M^{\prime}$ as follows. The machine $M^{\prime}$ first computes the formulas $g_{a, b, c}^{d}(s)$. For every Boolean variable $v$ in these formulas we introduce a new conjunct $\psi_{0}\left(\bar{x}_{v}\right) \vee \psi_{1}\left(\bar{x}_{v}\right)$ where $\bar{x}_{v}$ is a $d$-tuple of fresh variables and $\psi_{0}$ and $\psi_{1}$ are the two formulas defined in Lemma 6 Then, every positive literal $v$ in the original conjuncts of the formula is replaced by $\psi_{1}\left(\bar{x}_{v}\right)$, and every negative literal $l=\neg v$ by $\psi_{0}\left(\bar{x}_{v}\right)$. We then existentially quantify over all variables except for $\bar{x}_{z_{1}}, \ldots, \bar{x}_{z_{p}}$. Let $\psi_{a, b, c}^{d}(s)$ denote the resulting existential positive formula. For positive integers $k$ and $i$, we denote as $k[i]$ the $i$-th bit in the binary representation of $k$. Let $n$ be the total number of variables in the CSP instance $M_{w}(s)$ (in particular, $n \leq t$ ). It is clear that the formula

$$
\exists y_{1}, \ldots, y_{n}, \bar{x}_{z_{1}}, \ldots, \bar{x}_{z_{p}} . \bigwedge_{1 \leq a, k_{1}, \ldots, k_{l} \leq t}\left(\left(\bigwedge_{b \leq l, c} \psi_{a, b, c}^{k_{b}[c]}(s)\right) \rightarrow R\left(y_{k_{1}}, \ldots, y_{k_{l}}\right)\right)
$$

can be re-written in existential positive form $\Phi$ without blow-up: we can replace implications $\alpha \rightarrow \beta$ by $\neg \alpha \vee \beta$, and then move the negation to the atomic level, where we can remove negation by exchanging the role of $\varphi_{0}$ and $\varphi_{1}$. Hence, $\Phi$ can be computed by $M^{\prime}$ in polynomial time.

We claim that the formula $\Phi$ is true in $\Gamma$ if and only if there exists a computation of $M$ on $s$ that computes a satisfiable instance of $\operatorname{CSP}(\Gamma)$. To see this, let $w$ be a sufficiently long bit-string such that $M_{w}(s)$ is a satisfiable instance of $\operatorname{CSP}(\Gamma)$. Suppose for the sake of notation that the $n$ variables in $M_{w}(s)$ are the variables $y_{1}, \ldots, y_{n}$. Let $a_{1}, \ldots, a_{n}$ be a satisfying assignment to those $n$ variables. Then, if for $1 \leq i \leq n$ the variable $y_{i}$ in the formula $\Phi$ is set to $a_{i}$, and for $1 \leq i \leq p$ the variables $\bar{x}_{z_{i}}$ are set to a tuple that satisfies $\psi_{d}$ where $d$ is the $i$-th bit in $w$, we claim that the inner part of $\Phi$ is true in $\Gamma$. The reason is that, due to the way how we set the variables of the form $\bar{x}_{z_{i}}$, the precondition $\left(\bigwedge_{b \leq l, c} \psi_{a, b, c}^{k_{b}[c]}(s)\right)$ is true if and only if $R\left(y_{k_{1}}, \ldots, y_{k_{l}}\right)$ is a constraint in $M_{w}(s)$. Therefore, all the atomic formulas of the form $R\left(y_{k_{1}}, \ldots, x_{k_{l}}\right)$ are satisfied due to the way how we set the variables $y_{i}$, and hence $\Phi$ is true in $\Gamma$. It is straightforward to verify that the opposite implication holds as well, and this shows the claimed equivalence. 


\section{Structures With Function Symbols}

In this section, we briefly discuss the complexity of $\operatorname{ExPOS}(\Gamma)$ when $\Gamma$ might also contain functions. That is, we assume that the signature of $\Gamma$ consists of a finite set of relation and function symbols, and that the input formulas for the problem $\operatorname{ExPOS}(\Gamma)$ are existential positive first-order formulas over this signature. It is easy to see from the proofs in the previous section that when $\Gamma$ is not locally refutable, then $\operatorname{ExPOS}(\Gamma)$ is still NP-hard (with the same definition of local refutability as before).

The case when $\Gamma$ is locally refutable becomes more intricate when $\Gamma$ has functions. We present an example of a locally refutable structure $\Gamma$ where $\operatorname{ExPOS}(\Gamma)$ is NP-hard. Let the signature of $\Gamma$ be the structure $\left(2^{\mathbb{N}} ; \neq, \cap, \cup, c, \mathbf{0}, \mathbf{1}\right)$ where $\neq$ is the binary disequality relation, $\cap$ and $\cup$ are binary functions for intersection and union, respectively, $c$ is a unary function for complementation, and $\mathbf{0 , 1}$ are constants (i.e., 0 -ary functions) for the empty set and the full set $\mathbb{N}$, respectively.

Proposition 9 The structure $\left(2^{\mathbb{N}} ; \neq, \cap, \cup, c, \mathbf{0}, \mathbf{1}\right)$ is locally refutable.

Proof: By Lemma 6 is suffices to show that if $\Psi$ is a conjunction of atomic formulas that are satisfiable in $\Gamma$, then $\Psi$ is satisfiable over $\Gamma$. Since the only relation symbol in the structure is $\neq$, every conjunct in $\Psi$ is of the form $t_{1} \neq t_{2}$, where $t_{1}$ and $t_{2}$ are terms formed by variables and the function symbols $\cap, \cup, c, \mathbf{1}$ and $\mathbf{0}$. By Boole's fundamental theorem of Boolean algebras, $t=t^{\prime}$ can be re-written as $t^{\prime \prime}=0$. Therefore, $\Psi$ can be written as $t_{1} \neq \mathbf{0} \wedge \cdots \wedge t_{n} \neq \mathbf{0}$. Since $\Gamma$ is an infinite Boolean algebra, Theorem 5.1 in [7] shows that if $t_{i} \neq \mathbf{0}$ is satisfiable in $\Gamma$ for all $i \leq n$, then $\Psi$ is satisfiable in $\Gamma$ as well.

Proposition 10 The problem $\operatorname{ExPOS}\left(2^{\mathbb{N}} ; \neq, \cap, \cup, c, \mathbf{0}, \mathbf{1}\right)$ is NP-hard.

Proof: The proof is by reduction from SAT. Given a Boolean formula $\Psi$ in CNF with variables $x_{1}, \ldots, x_{n}$, we replace each conjunction in $\Psi$ by $\cap$, each disjunction by $\cup$, and each negation by $c$. Let $t$ be the resulting term over the signature $\{\cap, \cup, c\}$ and variables $x_{1}, \ldots, x_{n}$. It is easy to verify that $\exists x_{1}, \ldots, x_{n} . t \neq \mathbf{0}$ is true in $\Gamma$ if and only if $\Psi$ is a satisfiable Boolean formula.

\section{Conclusion}

In this paper, we proved that for an arbitrary (finite or infinite) relational structure the problem $\operatorname{ExPOS}(\Gamma)$ is in LOGSPACE if $\Gamma$ is locally refutable, or otherwise complete for the class $\operatorname{CSP}(\Gamma)_{\mathrm{NP}}$ under deterministic polynomial-time many-one reductions. In particular, if $\Gamma$ is not locally refutable then the problem $\operatorname{ExPOS}(\Gamma)$ is NP-hard. Structures with a finite domain are locally refutable if and only if they are $a$-valid for some value $a$ of the domain $D$. Finally, we present an example of a structure that shows that our result cannot be straightforwardly extended to structures $\Gamma$ with function symbols, since local refutability of $\Gamma$ no longer implies that $\operatorname{ExPOS}(\Gamma)$ is in LOGSPACE when $\Gamma$ contains function symbols.

\section{Acknowledgment}

We would like to thank Víctor Dalmau for helpful suggestions, and Moritz Müller for the encouragement to study the case where the structure $\Gamma$ contains function symbols. 


\section{References}

[1] N. Creignou, Ph. G. Kolaitis, and H. Vollmer, editors. Complexity of Constraints - An Overview of Current Research Themes, volume 5250 of Lecture Notes in Computer Science, Springer Verlag, 2008.

[2] M. Bodirsky and M. Grohe. Non-Dichotomies in Constraint Satisfaction Complexity. Proceedings 35th International Colloquium on Automata, Languages and Programming (ICALP 2008), Part II, Reykjavik (Iceland), volume 5126 of Lecture Notes in Computer Science, 184-196, 2008.

[3] M. Bodirsky, M. Hermann and F. Richoux. Complexity of Existential Positive First-Order Logic. Proceedings 5th Conference on Computability in Europe (CiE 2009), Heidelberg (Germany), 31-36, 2009.

[4] M. R. Garey and D. S. Johnson. Computers and Intractability: A Guide to the Theory of NPCompleteness. W.H. Freeman and Co, 1979.

[5] M. Hermann and F. Richoux. On the Computational Complexity of Monotone Constraint Satisfaction Problems. Proceedings 3rd Annual Workshop on Algorithms and Computation (WALCOM 2009), Kolkata (India), 286-297, 2009.

[6] R. E. Ladner, N. A. Lynch and A. L. Selman. A Comparison of Polynomial-Time Reducibilities. Theoretical Computer Science, 1(2), 103-124, 1975.

[7] K. Marriott and M. Odersky. Negative boolean constraints. Theoretical Computer Science, 160(1\&2), 365-380, 1996.

[8] B. Martin. Dichotomies and Duality in First-order Model Checking Problems. CoRR abs/cs/0609022, 2006.

[9] B. Martin. First-Order Model Checking Problems Parameterized by the Model. Proceedings 4th Conference on Computability in Europe (CiE 2008), Athens (Greece), 417-427, 2008. 